\title{
Regional Analysis of the Magnetization Transfer Ratio of the Brain in Mild Alzheimer Disease and Amnestic Mild Cognitive Impairment
}

\author{
M. Mascalchi, A. Ginestroni, V. Bessi, N. Toschi, S. Padiglioni, S. Ciulli, C. Tessa, M. Giannelli, L. Bracco, and S. Diciotti
}

\begin{abstract}
BACKGROUND AND PURPOSE: Manually drawn VOI-based analysis shows a decrease in magnetization transfer ratio in the hippocampus of patients with Alzheimer disease. We investigated with whole-brain voxelwise analysis the regional changes of the magnetization transfer ratio in patients with mild Alzheimer disease and patients with amnestic mild cognitive impairment.
\end{abstract}

MATERIALS AND METHODS: Twenty patients with mild Alzheimer disease, 27 patients with amnestic mild cognitive impairment, and 30 healthy elderly control subjects were examined with high-resolution TIWI and 3-mm-thick magnetization transfer images. Whole-brain voxelwise analysis of magnetization transfer ratio maps was performed by use of Statistical Parametric Mapping 8 software and was supplemented by the analysis of the magnetization transfer ratio in FreeSurfer parcellation-derived VOls.

RESULTS: Voxelwise analysis showed 2 clusters of significantly decreased magnetization transfer ratio in the left hippocampus and amygdala and in the left posterior mesial temporal cortex (fusiform gyrus) of patients with Alzheimer disease as compared with control subjects but no difference between patients with amnestic mild cognitive impairment and either patients with Alzheimer disease or control subjects. VOI analysis showed that the magnetization transfer ratio in the hippocampus and amygdala was significantly lower (bilaterally) in patients with Alzheimer disease when compared with control subjects (ANOVA with Bonferroni correction, at $P<$ .05). Mean magnetization transfer ratio values in the hippocampus and amygdala in patients with amnestic mild cognitive impairment were between those of healthy control subjects and those of patients with mild Alzheimer disease. Support vector machine-based classification demonstrated improved classification performance after inclusion of magnetization transfer ratio-related features, especially between patients with Alzheimer disease versus healthy subjects.

CONCLUSIONS: Bilateral but asymmetric decrease of magnetization transfer ratio reflecting microstructural changes of the residual GM is present not only in the hippocampus but also in the amygdala in patients with mild Alzheimer disease.

ABBREVIATIONS: AD = Alzheimer disease; $\mathrm{AUC}=$ area under the receiver operating characteristic curve; DARTEL $=$ Diffeomorphic Anatomical Registration through Exponentiated Lie Algebra; $\mathrm{MCl}=$ mild cognitive impairment; $\mathrm{MT}=$ magnetization transfer

$A_{t}$ lzheimer disease $(\mathrm{AD})$ is the most common cause of dementia and a major cause of morbidity worldwide. ${ }^{1}$ In recent years, considerable efforts have been made to better understand

Received December 12, 2012; accepted after revision February 9, 2013

From the Quantitative and Functional Neuroradiology Research Unit, Department of Experimental and Clinical Biomedical Sciences (M.M., A.G., S.C., S.D.), and Department of Neurological and Psychiatric Sciences (V.B., S.P., L.B.), University of Florence, Florence, Italy; Medical Physics Section (N.T.), Faculty of Medicine, University of Rome "Tor Vergata," Rome, Italy; Radiology Department (C.T.), Versilia Hospital, Lido di Camaiore (Lucca), Italy; and Unit of Medical Physics (M.G.), Pisa University Hospital, “Azienda Ospedaliero-Universitaria Pisana," Pisa, Italy.

Please address correspondence to Mario Mascalchi, MD, PhD, Quantitative and Functional Neuroradiology Research Unit, Department of Experimental and Clinical Biomedical Sciences, University of Florence, Viale Morgagni 85, Florence, Italy; e-mail: m.mascalchi@dfc.unifi.it

Indicates article with supplemental on-line figures

Evidence-Based Medicine Level 2. the natural evolution of $\mathrm{AD}$, with particular focus on the presymptomatic and early symptomatic phases of the disease, whose characterization could enable earlier and potentially more effective treatment. These efforts have led to identification of a heterogeneous condition called mild cognitive impairment (MCI), which is characterized by objective evidence of cognitive decline without deficit in daily living activities. ${ }^{2}$ In particular, it is assumed that a particular form of MCI, termed amnestic MCI, can represent an intermediary predementia stage of $\mathrm{AD} .^{3}$

The search for biomarkers that may reflect specific in vivo features of pathophysiologic processes underlying $\mathrm{AD}$ is drawing increasing attention and financial resources. ${ }^{1}$

MRI allows in vivo investigation of the structural brain changes in $\mathrm{AD} .{ }^{4} \mathrm{~T} 1 \mathrm{WI}$ shows global and local volume loss in

http://dx.doi.org/10.3174/ajnr.A3568 
structures typically affected by the disease such as the temporal lobe, including the hippocampus, amygdala, and enthorinal cortex. ${ }^{5-8}$ DTI and magnetization transfer (MT) imaging allow assessment of the microstructure of the residual WM and GM in the same structures. ${ }^{9-16}$ In particular, MT is a peculiar MR contrast that is based on the exchange of magnetization between free protons and protons bound to macromolecules and is influenced by the direct saturation of free protons by the radiofrequency pulse. ${ }^{14,16}$ A more comprehensive analysis of the magnetization transfer phenomenon is possible with a quantitative approach, which, by use of different magnetization transfer models, enables determination of several parameters including the relative size of the restricted proton pool, the $\mathrm{T} 2$ relaxation time of the restricted pool and the relaxation times T1 and T2 of the free pool, and the forward exchange rate. ${ }^{14-16}$

In its simplest version, MT is obtained with acquisition of a pair of images without and with a specific radiofrequency offresonance prepulse followed by image subtraction and computation of the MT ratio. MT ratio is decreased in the temporal lobe and hippocampus of patients with $\mathrm{AD}$ independent from atrophy $^{9-12,14,17,18}$; is variably correlated with clinical severity, neuropsychological measurements, and disease duration ${ }^{9-12}$; and appears capable to track progression of regional GM microstructural changes in $\mathrm{AD} .{ }^{18} \mathrm{MT}$ ratio was also reported to be decreased in the whole brain, whole temporal lobe, or temporal lobe GM and WM in patients with amnestic MCI as compared with agematched healthy control subjects. ${ }^{10,11,19,20}$ However, in 2 studies that compared the MT ratio with several parameters calculated with quantitative MT approach in the hippocampus, enthorinal cortex, insula, and temporal neocortex, the former delivered the worst performance in differentiating $\mathrm{AD}, \mathrm{MCI}$, or healthy control subjects. ${ }^{14,16}$

All the above MT imaging studies in AD and MCI used whole brain or temporal lobe segmentation, or involved analysis of manually drawn region of interest or VOI. Voxelwise analysis of MT ratio maps offers the advantage of an automatic whole-brain, voxel-based analysis without a priori assumptions about the specific region under investigation. ${ }^{21}$ Thus far, this approach was applied in only 1 study that evaluated several sophisticated parameters calculated from quantitative MT imaging but not MT ratio and reported significantly reduced efficiency of the transfer of magnetization between the 2 pools in the hippocampus, temporal lobe, posterior cingulate, and posterior parietal cortex of patients with $\mathrm{AD}$ compared with healthy control subjects. ${ }^{15}$

In the present study, we evaluated the MT ratio with a voxelwise, whole-brain approach supplemented by automatic VOI analysis in a group of patients with mild $\mathrm{AD}$, a group of patients with amnestic MCI, and a group of healthy elderly control subjects. The 2 aims of our study were 1 ) to map the regional distribution of MT ratio in mild $\mathrm{AD}$ and 2) to further explore the regional changes in $\mathrm{MT}$ ratio in amnestic MCI.

\section{MATERIALS AND METHODS \\ Subjects}

The study was based on 47 subjects referred to the memory clinic of the University of Florence who were consecutively observed over a period of 2 years and evaluated through an extensive standardized neuropsychological battery. ${ }^{22}$

Twenty (17 women and 3 men; mean age, $74.4 \pm 7.0$ years; range, 59-85 years) fulfilled the NINCDS-ADRDA criteria for probable $\mathrm{AD},{ }^{23}$ and, on the basis of the results of the Mini-Mental State Examination (mean, $25 \pm 2.5$; range, 21.3-30), received a diagnosis of mild AD.

Twenty-seven (15 women and 12 men; mean age, $68.8 \pm 7.8$ years; range, 51-82 years) fulfilled the Winblad et $\mathrm{al}^{2}$ criteria for amnestic MCI. In particular, they were judged as both not normal and not fulfilling diagnostic criteria for dementia (Diagnostic and Statistical Manual of Mental Disorders IV, ICD 10). Moreover, they had preserved basic activities of daily living or minimal impairment in complex instrumental functions in combination with evidence of memory decline, measured either by self-report and/or informant report in conjunction with deficits on objective cognitive tasks and/or evidence of memory decline over time on objective neuropsychological tests. Their mean Mini-Mental State Examination score was $27.9 \pm 1.8$ (range, 23-30).

Thirty healthy subjects (18 women and 12 men; mean age, $71.9 \pm 6.1$ years; range, $57-82$ ) without familial or personal history of neurologic or psychiatric disorders served as control subjects. They underwent a neurologic examination that showed no abnormalities. Their mean Mini-Mental State Examination score was $28.8 \pm 1.2$ (range, 26.2-30).

The 3 groups of subjects were matched for sex $\left(\chi^{2}, P=.12\right)$. Patients with $\mathrm{AD}$ and healthy control subjects as well as patients with MCI and healthy control subjects did not differ significantly in terms of mean age, whereas the patients with MCI were younger than the patients with $\mathrm{AD}$ (ANOVA with Bonferroni correction, with $P<.05)$.

\section{MRI Protocol}

Within 3 months of the clinical evaluation and recruitment, patients and control subjects underwent MRI examination on a 1.5T system (Intera; Philips, Best, the Netherlands) with $33 \mathrm{mT} / \mathrm{m}$ maximum gradient strength and a 6-channel head coil. After the scout image, a sagittal 3D T1-weighted turbo gradient-echo sequence $\left(\mathrm{TR}=8.1 \mathrm{~ms}, \mathrm{TE}=3.7 \mathrm{~ms}\right.$, flip angle $=8^{\circ}, \mathrm{TI}=764 \mathrm{~ms}$, $\mathrm{FOV}=256 \times 256 \mathrm{~mm}$, matrix size $=256 \times 256,160$ contiguous sections, section thickness $=1 \mathrm{~mm}, \mathrm{NEX}=1$, acceleration factor (SENSE) $=2$ ) was acquired for quantitative volumetric assessment of GM and as a high-resolution anatomic reference of MT images. For MT imaging, axial images without (M0) and with (Ms) gaussian sinc-shaped off-resonance pulse (bandwidth $=342$ $\mathrm{Hz}$, offset frequency $=1100 \mathrm{~Hz}$, duration $=17,500 \mu \mathrm{s}$ ) were acquired with a gradient recalled-echo sequence $(\mathrm{TR}=37 \mathrm{~ms}$, $\mathrm{TE}=3.7 \mathrm{~ms}$, flip angle $=8^{\circ}, \mathrm{FOV}=256 \times 256 \mathrm{~mm}$, matrix size $=$ $128 \times 128,100$ contiguous sections, section thickness $=3 \mathrm{~mm}$, $\mathrm{NEX}=2$, acceleration factor $($ SENSE) $=2$ ). A coronal FLAIR sequence $(\mathrm{TR}=11,000 \mathrm{~ms}, \mathrm{TE}=140 \mathrm{~ms}, \mathrm{TI}=2800 \mathrm{~ms}, \mathrm{FOV}=$ $230 \mathrm{~mm}$, matrix size $=320 \times 216$, contiguous sections, section thickness $=5 \mathrm{~mm}$ ) was also performed for evaluation of nonspecific T2 hyperintensities of the cerebral WM observed in elderly subjects, termed leukoaraoisis.

The acquisition time for the entire MR protocol was approximately 17 minutes, with 8 minutes required for MT imaging.

AJNR Am J Neuroradiol 34:2098-104 Nov 2013 www.ajnr.org 


\section{T2 Hyperintensities of the Cerebral WM}

One operator (M.M.) with 25 years of experience in clinical MRI who was blinded to the clinical data evaluated the FLAIR images of all subjects to rate the extension of cerebral leukoaraoisis by use of the $0-3$ visual scale proposed by Fazekas et al. ${ }^{24}$

\section{MT Ratio Map Computation}

For each subject, after $6 d f$ co-registration of M0-Ms images by use of FLIRT (FMRIB's Linear Image Registration Tool; http:// www.fmrib.ox.ac.uk/fsl), ${ }^{25}$ the MT ratio map was calculated, voxel by voxel, according to the following formula $[(\mathrm{M} 0-\mathrm{Ms}) /$ M0] $\times 100$. A brain mask was also computed by use of the FSL Brain Extraction Tool on the Ms image. Values of MT ratio map $>75$, assumed to be caused by image noise or partial volume effects or outside the brain mask, were replaced by zeroes. ${ }^{26}$

\section{Voxel-Based Morphometry}

Preprocessing of the T1WI was performed by use of the Statistical Parametric Mapping 8 package (http://www.fil.ion.ucl.ac.uk/ spm) and the Voxel Based Morphometry 8 toolbox (http:// dbm.neuro.uni-jena.de). ${ }^{27}$ All T1WI was corrected for bias-field inhomogeneities, then spatially normalized to the standard Diffeomorphic Anatomical Registration through Exponentiated Lie Algebra (DARTEL) T1 template in Montreal Neurological Institute space by means of linear and nonlinear transformations and segmented into GM, WM, and CSF within the same generative model. ${ }^{28}$ The segmentation procedure was further extended by accounting for partial volume effects, ${ }^{29}$ by applying adaptive maximum a posteriori estimations, ${ }^{30}$ and by use of a hidden Markov random field model, ${ }^{31}$ as described previously. ${ }^{32}$ The resulting GM and WM images were modulated to account for volume changes resulting from the normalization process. We considered only nonlinear volume changes so that further analyses did not have to account for differences in head size. Finally, images were smoothed with a gaussian kernel of $8 \mathrm{~mm}$ (full width at half maximum).

Voxelwise GM and WM differences between patients with $\mathrm{AD}$ and patients with MCI and control subjects were examined by means of 1-way ANOVA with age and sex as nuisance variables. To avoid possible edge effects between different tissue types, we excluded all voxels with GM or WM tissue probability values of $<.1$ (absolute threshold masking). We applied a statistical threshold of $P<.05$ with family-wise error rate correction.

\section{Voxelwise MT Ratio Analysis}

For each subject, the Ms image was affinely co-registered with a 12 $d f$ transformation to the raw T1WI, and this transformation was applied to the MT ratio map. The MT ratio map was then normalized to the Montreal Neurological Institute space by means of the transformation previously computed when co-registering the raw T1WI to the standard DARTEL T1 template. Finally, voxelwise MT ratio differences between patients with $\mathrm{AD}$ and patients with $\mathrm{MCI}$ and control subjects were examined by means of 1-way ANOVA, with age and sex as nuisance variables. We applied a statistical threshold of $P<.05$ with family-wise error rate correction.

\section{VOI-Based MT Ratio Analysis}

Completely automated subcortical reconstruction and volumetric segmentation of each subject's structural T1-weighted MRI scan were performed with the FreeSurfer image analysis suite (http://surfer.nmr.mgh.harvard.edu/). ${ }^{33}$ Briefly, this includes removal of nonbrain tissue by use of a hybrid watershed/surface deformation procedure, automated Talairach transformation, segmentation of the subcortical WM and deep GM volumetric structures, intensity normalization, tessellation of the GM/WM boundary, automated topology correction, and surface deformation after intensity gradients to optimally place the GM/WM and GM/CSF borders at the location where the greatest shift in intensity defines the transition to the other tissue class. Volumetric regions of interest delineating left and right hippocampus and amygdala in each subject's native T1 space were obtained by affine transformation. To reduce partial volume effects, each VOI was eroded with a $3 \mathrm{D}$ structural element with $1-\mathrm{mm}$ radius. VOIs in the amygdala containing a small $\left(<150 \mathrm{~mm}^{3}\right.$ of volume $)$ number of voxels were excluded from further analyses. One operator (A.G.), blinded to the clinical data, visually judged the VOIs automatically placed in the hippocampus and amygdala as adequate or inadequate. In particular, he excluded the VOI with evident CSF contamination.

For each subject, the affine transformation from T1WI to the Ms image was computed as the inverse of the previously computed affine transformation from the Ms image to the T1WI. After erosion, the transformation from T1WI to the Ms image was applied to each VOI, and the mean MT ratio was determined in this transformed VOI.

The comparison of mean MT ratio within the hippocampus and amygdala VOIs in the healthy control subjects, patients with $\mathrm{MCI}$, and patients with $\mathrm{AD}$ was performed by means of ANOVA test with post hoc Bonferroni correction, with $P<.05$.

\section{Classification by Means of Support Vector Machines}

With the goal of exploring the discriminative power of automatic VOI analysis of MT ratio in the hippocampus and amygdala, we trained a popular machine learning scheme, that is, a support vector machine (with linear kernel and complexity parameter $C=$ 1), implemented through a sequential minimal optimization algorithm. ${ }^{34}$ In this context, we considered only the subset of patients for which the complete set of hippocampus and amygdala VOI measurements was available. We took into account the imbalance in the number of subjects within the different classes by the cost matrix method. ${ }^{34}$ We studied 2 different classification tasks: 1) patients with $\mathrm{AD}$ patients versus healthy subjects and 2) patients with AD versus with MCI. For each classification task, we initially fed the classifier with the VOI volumes only, and successively with both VOI volumes and VOI MT ratios. To fully explore the solution space in a rigorous manner, we performed an exhaustive search on the different feature vectors by use of a 10 -fold cross-validation technique for training and testing the classifiers. Performances were evaluated through the area under receiver operating characteristic curve (AUC), in which highest values of AUC were considered better, and, in the case of equal ranking, the result obtained with a lower number of features was considered better. Sensitivity and specificity were also recorded. 

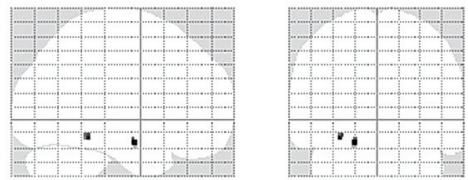

A

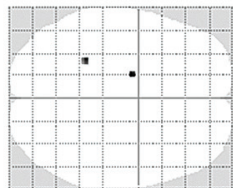

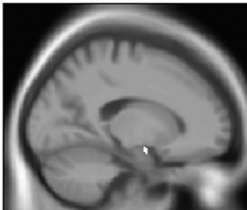

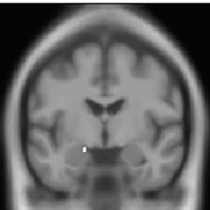

B
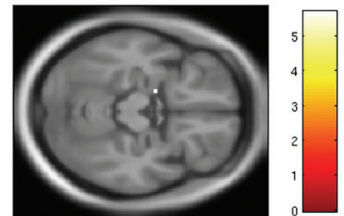

FIG 1. SPM8 "glass brain" representation (A) of voxelwise analysis between control subjects and patients with Alzheimer disease showing significant clusters of decreased magnetization transfer ratio in the left hippocampus and amygdala and in the posterior mesial temporal cortex (fusiform gyrus) $(P<.05$, with family-wise error rate correction). Superimposition onto $T 1$ template of the cluster in the hippocampus and amygdala are demonstrated in $B$ and of the cluster in the fusiform gyrus in $C$.
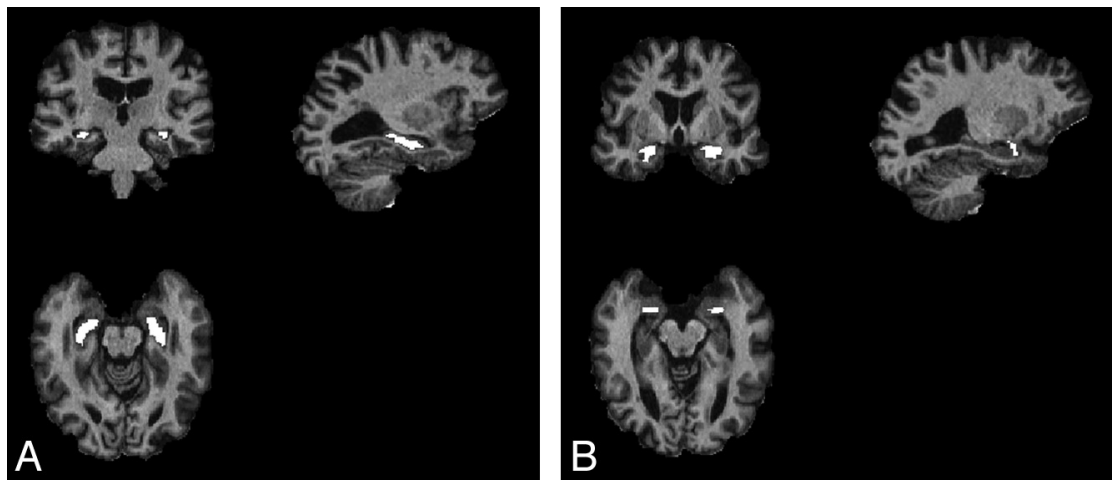

FIG 2. Example of the FreeSurfer automatic VOI segmentation of the hippocampus (A) and amygdala (B) eroded with a 3D structural element with 1-mm radius.

All classification tasks were performed with the use of WEKA software (http://www.cs.waikato.ac.nza/mL/weka, version 3.6.8). ${ }^{34}$

\section{RESULTS}

\section{T2 Hyperintensities of the Cerebral WM}

Extension of leukoaraoisis was similar in the 3 groups of subjects (mean in AD $1.00 \pm 0.56$; mean in amnestic MCI, $0.92 \pm 0.61$; mean in control subjects, $1.07 \pm 0.58$ [a nonsignificant difference at the Kruskal-Wallis test, $P=.66]$ ).

\section{Regional Volume}

Voxel-based morphometry $(P<.05$, ANOVA with family-wise error rate correction for multiple comparisons) showed 2 isolated clusters of cortical GM atrophy in the right $(x=28 \mathrm{~mm}, y=-27$ $\mathrm{mm}, z=-5 \mathrm{~mm})$ and left $(x=-28 \mathrm{~mm}, y=-30 \mathrm{~mm}, z=-8$ $\mathrm{mm}$ ) hippocampus and parahippocampal gyrus, which appeared more extensive on the right side, in patients with $\mathrm{AD}$ as compared with healthy control subjects (On-line Fig 1) and 1 isolated cluster $(x=-15 \mathrm{~mm}, y=56 \mathrm{~mm}, z=15 \mathrm{~mm})$ in the left superior frontal gyrus in patients with amnestic MCI as compared with healthy control subjects (On-line Fig 2). No significant clusters were observed in patients with $\mathrm{AD}$ as compared with patients with amnestic MCI. No significant regional decrease of WM volume was observed in the 2 groups of patients with respect to each other and the control group.

\section{Regional MT Ratio}

Voxelwise analysis showed 2 circumscribed clusters of decreased MT ratio, 1 located in the left hippocampus and amygdala $(x=-16 \mathrm{~mm}, y=-6 \mathrm{~mm}$, $z=-17 \mathrm{~mm}$ ) and 1 in the posterior mesial temporal cortex (fusiform gyrus) $(x=-28 \mathrm{~mm}, y=-40 \mathrm{~mm}, z=-14$ $\mathrm{mm}$ ) of patients with $\mathrm{AD}$ as compared with control subjects and no difference between patients with $\mathrm{AD}$ and patients with amnestic MCI or between patients with MCI and control subjects (Fig 1).

\section{Automatic VOI Analysis}

Fig 2 shows examples of the automatic VOIs obtained by use of FreeSurfer. Six ( 3 in patients with AD, 1 in a patient with MCI, and 2 in healthy subjects) of $144 \mathrm{VOIs}$ were judged to partially fall outside the hippocampus, whereas 26 ( 9 in patients with $\mathrm{AD}, 5$ in patients with MCI, and 12 in healthy subjects) of 144 VOIs in the amygdala were constituted by a number of voxels that was considered too small to afford a reliable evaluation of that structure.

The MT ratio in the bilateral hippocampus and amygdala was significantly lower in patients with $\mathrm{AD}$ when compared with control subjects (ANOVA with post hoc Bonferroni correction, with $P<.05$ ) (Fig 3).

The values of the mean MT ratio in hippocampus and amygdala in the patients with amnestic MCI were between those of healthy control subjects and those of patients with mild AD (Fig 3 ), and the differences were not significant with the exception of the MT ratio in the left amygdala, which was significantly lower in $\mathrm{AD}$ than in amnestic MCI.

\section{Classification by Use of Support Vector Machines}

The classification tasks were performed on the subset of subjects for which the complete set of hippocampus and amygdala VOI measurements were available (ie, 23 healthy subjects, 22 patients with MCI, and 12 patients with $\mathrm{AD}$ ).

In the patients with $\mathrm{AD}$ versus control subjects classification task, the best feature vector was composed of the volumes of bilateral amygdala, right hippocampus, and of the MT ratio of left AJNR Am J Neuroradiol 34:2098-104 Nov 2013 www.ajnr.org 


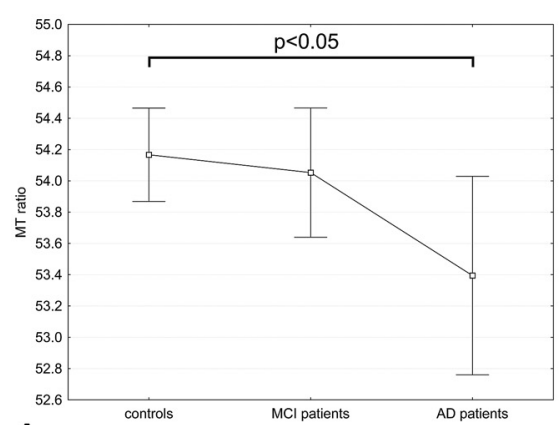

A
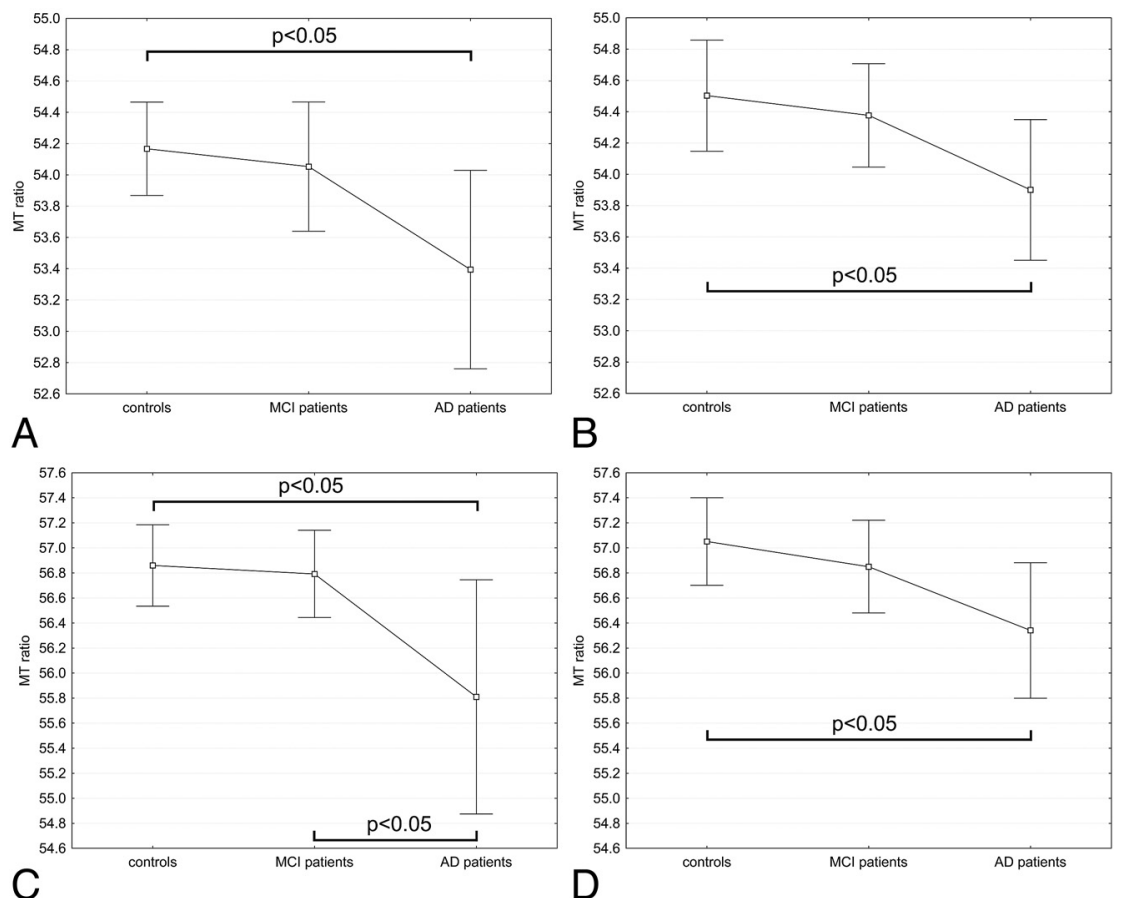

FIG 3. Magnetization transfer ratio of in the hippocampi [left (A) and right (B)] and amygdalae [left (C) and right (D)] in the 3 groups. Mean and 95\% confidence intervals of mean MT ratio are reported. The MT ratio in the bilateral hippocampus and amygdala was significantly lower in patients with Alzheimer disease when compared with control subjects (ANOVA with post hoc Bonferroni correction, with $P<.05$ ). The values of the mean MT ratio in the hippocampus and amygdala in the patients with amnestic mild cognitive impairment were between those of healthy control subjects and those of patients with mild AD, and the differences were not significant with the exception of the MT ratio in the left amygdala, which was significantly lower in $\mathrm{AD}$ than in amnestic $\mathrm{MCl}$.

hippocampus, reaching a 0.873 AUC, $83.3 \%$ sensitivity, and 95.7\% specificity. This result was markedly higher than the best performance obtained with volumetric features only (0.788 AUC, $75.0 \%$ sensitivity, and $82.6 \%$ specificity, obtained by using bilateral amygdalae and right hippocampus volumes).

In the $\mathrm{AD}$ versus MCI classification task, the best feature vector was composed of the volumes of right hippocampus, left amygdala, and MT ratio of left hippocampus and right amygdala (0.761 AUC, $75.0 \%$ sensitivity, and $77.3 \%$ specificity). This result was slightly better than the best performance obtained with volumetric features only (0.746 AUC, 58.3\% sensitivity, $90.9 \%$ specificity, and obtained by bilateral hippocampus).

\section{DISCUSSION}

Our investigation with the use of automatic whole-brain, voxelwise, and VOI analyses demonstrates that a decrease of MT ratio, presumably reflecting microstructural changes related to $\mathrm{AD}$ pathology, can be observed in patients with mild $\mathrm{AD}$, not only in the hippocampus but also in the amygdala bilaterally (whereas more markedly on the left side). In patients with amnestic MCI, the MT ratio in the same structures tends to be decreased as compared with healthy control subjects but less conspicuously than in patients with mild AD.

The decreased MT ratio revealed by voxelwise analysis in the left hippocampus and amygdala and in the left posterior mesial temporal cortex in patients with mild $\mathrm{AD}$ as compared with healthy control subjects confirms previous data obtained by means of manually drawn regions of interest or VOIs in the hippocampus $9,12,14,18$ and extends to the amygdala the capability of MT ratio to detect microstructural GM changes related to $\mathrm{AD}$ pathology.

The hippocampus and amygdala are among the earliest mesial temporal lobe structures affected by AD pathology along with transentorhinal regions, subiculum, and entorhinal cortex. ${ }^{35}$ Moreover, volumetry MRI data in vivo indicate that both the hippocampus and amygdala show global or local atrophy in $\mathrm{AD}^{5-8}$

The automatic VOI analysis in our study showed that the decrease of the MT ratio in the hippocampus and amygdala was prominent in the left side but was also significant in the right side.

Asymmetric distribution of pathologic changes ${ }^{36}$ and atrophy ${ }^{4,5}$ with an average predominance in the left temporal lobe side is a well-known feature of AD. However, cases with right-side predominance of $\mathrm{AD}$ pathologic changes were observed, ${ }^{37}$ and the results of an MRI study specifically assessing lateral distribution of atrophy in vivo ${ }^{38}$ justify the view that $\mathrm{AD}$ alterations are asymmetric but not lateralized. In all previous MT imaging studies in $\mathrm{AD}$ and $\mathrm{MCI}$, no side evaluation of lobar or regional MT ratio was reported, and data related to the 2 sides were typically averaged. ${ }^{9-12,14,18}$

In our study, the voxelwise analysis did not show any region exhibiting significantly different MT ratio in the group of patients with amnestic MCI with respect to healthy control subjects, and automatic VOI analysis of the hippocampus and amygdala in the patients with amnestic MCI showed values for the MT ratio that were between the values computed for healthy control subjects and patients with $\mathrm{AD}$. This result is in line with the results obtained with manual segmentation of the anterior hippocampus in a prior study, ${ }^{14}$ whereas it represents a partial discrepancy with prior investigations in which the MT ratio of the whole brain or temporal lobe was found to be significantly decreased in patients with MCI as compared with healthy control subjects. ${ }^{10,11,19,20}$

Considering the fact that our study involved the highest number of subjects with amnestic MCI examined with MT imaging so far, we submit the following 2 (not mutually exclusive) explanations for this discrepancy.

First, global analyses of the whole temporal lobe or of the temporal GM or WM have an inherently higher statistical power when compared with regional analyses that are based on either voxelwise techniques or smaller VOIs, because the former techniques imply computation of the variable of interest (in our case, 
MT ratio) in a far greater number of voxels. This aspect might also account for lack of significant changes of the MT ratio in other cortical GM regions and in the WM in patients with $\mathrm{AD}$ in the voxelwise analysis in our sample.

Second, MCI is a transitory heterogeneous condition, and progression of amnestic MCI to AD is observed only in a portion of subjects attending a memory clinic, namely, 9.6\% per year, as indicated in a meta-analysis. ${ }^{39}$ As a matter of fact, only approximately $60 \%$ of patients with amnestic MCI, termed converters, ultimately have development of probable $\mathrm{AD}$ after 5 years, whereas the remainder have other forms of dementia, remain stable, or even revert to normal. ${ }^{40}$ We submit that in our cohort, the paucity of patients with MCI who had development of AD during the 2 years successive to their recruitment in the MRI study ( 5 of 27 individuals; namely, 18.5\%) could justify the lack of significant changes of MT ratio in the patients with MCI considered as a group. Longitudinal clinical evaluation of our patients with MCI is underway to verify this hypothesis.

The latter explanation could also apply to the voxel-based morphometry results that we obtained. In fact, although the bilateral hippocampal atrophy observed in our patients with mild $\mathrm{AD}$ as compared with healthy control subjects is in line with all prior studies, ${ }^{4}$ we identified only a small cluster of decreased GM in the left superior frontal gyrus in the amnestic MCI group as compared with healthy normal control subjects, with no significant changes in the temporal lobe. Indeed, thus far, MRI volumetry has provided contradictory results in groups of patients with MCI (including converters and nonconverters) when compared with healthy control subjects. Several studies, by use of manual VOI positioning or voxel-based morphometry techniques, have indicated that patients with MCI as a group showed atrophy of mesial temporal structures similar to those characteristic of AD. ${ }^{5,41}$ However, no significant differences between patients with MCI and healthy elderly control subjects were reported in the mesial temporal lobe GM in voxel-based morphometry studies based on smaller cohorts that used restrictive statistical thresholds $s^{42,43}$ or that considered age and Mini-Mental State Examination as nuisance variables. ${ }^{44}$ Isolated decrease of GM volume in the left middle frontal gyrus was reported in a previous voxelbased morphometry study when comparing patients with amnestic MCI and healthy control subjects. ${ }^{43}$

It is important to note that in both classification tasks, the exhaustive search demonstrated that the best feature vector included the MT ratio value of 1 or 2 VOIs. In particular, we observed that including MT ratio-related features resulted in improved detection performance, both when discriminating patients with $\mathrm{AD}$ from healthy subjects (albeit to a lesser extent) and when discriminating patients with $\mathrm{AD}$ from patients with MCI. These results demonstrate that MT ratio-related features provide added value with improvement in classification performance, especially between patients with $\mathrm{AD}$ versus healthy subjects. This supports the inclusion of MT ratio in more articulate machine learning-based studies for discrimination of patients with $\mathrm{AD}$.

We can only speculate about the pathologic substrate of the decreased MT ratio in the hippocampus and amygdala that we observed in patients with mild $\mathrm{AD}$. It presumably reflects differ- ences in macromolecular tissue composition associated with local accumulation of amyloid plaques, neurofibrillary tangles, or microglia cells, as observed in early AD. ${ }^{35}$ In particular, a reduced capacity to exchange magnetization as the result of the presence of amyloid $\beta$ plaques (a non-H-bonding group) through a surfacehydrophobicity effect was hypothesized as a possible mechanism underlying the decrease of MT ratio in AD. ${ }^{14,15}$

We recognize 2 main limitations of our study. First, we obtained source MT images with a relatively coarse spatial resolution as compared with T1WI. Three-dimensional sequences for MT imaging are now available and enable acquisition of images with increased spatial resolution, ${ }^{45}$ especially in combination with higher magnetic field strength scanners. This improved spatial resolution of MT images could be valuable for VOI analysis of MT ratio in the cerebral gyri in which voxelwise analysis showed significantly decreased MT. Such an analysis could not be performed in the present investigation because of the small number of voxels that survived erosion in cortical GM.

Second, we investigated the crude MT ratio. More sophisticated quantitative analyses of MT effect in the whole or anterior hippocampus were successfully applied to the differentiation of patients with $\mathrm{AD}$, patients with $\mathrm{MCI}$, and healthy control subjects, ${ }^{13,16}$ but they require longer acquisition times and complex data modeling.

\section{CONCLUSIONS}

Bilateral but asymmetric decrease of MT ratio reflecting microstructural changes of the residual GM is present not only in the hippocampus but also in the amygdala in patients with mild AD. Besides volume loss, regional decrease in MT ratio may contribute to MRI-based classification of single patients presenting with a memory complaint.

\section{REFERENCES}

1. Henry MS, Passmore AP, Todd S, et al. The development of effective biomarkers for Alzheimer's disease: a review. Int J Geriatr Psychiatry 2013;28:331-40.

2. Winblad B, Palmer K, Kivipelto M, et al. Mild cognitive impairment-beyond controversies, towards a consensus: report of the International Working Group on Mild Cognitive Impairment. J Intern Med 2004;256:240-46

3. Petersen RC, Trojanowski JQ. Use of Alzheimer disease biomarkers: potentially yes for clinical trials but not yet for clinical practice. JAMA 2009;302:436-37

4. Ramani A, Jensen JH, Helpern JA. Quantitative MR imaging in Alzheimer's disease. Radiology 2006;241:26-43

5. Risacher SI, Saykin AJ, West JD, et al. Baseline MRI predictors of conversion from MCI to probable AD in the ADNI Cohort. Curr Alzheimer Res 2009;6:347-61

6. Frisoni GB, Ganzola R, Canu E, et al. Mapping local hippocampal changes in Alzheimer's disease and normal ageing with MRI at 3 Tesla. Brain 2008;131:3266-76

7. Cavedo E, Boccardi M, Ganzola R, et al. Local amygdala structural differences with 3T MRI in patients with Alzheimer disease. Neurology 2011;76:726-33

8. Poulin SP, Dautoff R, Morris JC, et al. Amygdala atrophy is prominent in early Alzheimer's disease and relates to symptom severity. Psychiatr Res Neuroimaging 2011;194:7-13

9. Hanyu H, Asano H, Sakurai M, et al. Magnetization transfer measurements of the hippocampus in the early diagnosis of Alzheimer's disease. J Neurol Sci 2001;188:79-84 
10. van der Flier WM, van den Heuvel DM, Weverling-Rijnsburger AW, et al. Magnetization transfer imaging in normal aging, mild cognitive impairment, and Alzheimer's disease. Ann Neurol 2002;52:62-67

11. Van Es ACGM, van der Flier WM, Admiraal Behloul F, et al. Lobar distribution of changes in gray matter and white matter in memory clinic patients: detected using magnetization transfer imaging. AJNR Am J Neuroradiol 2007;28:1938-42

12. Ridha BH, Symms MR, Tozer DJ, et al. Magnetization transfer ratio in Alzheimer disease: comparison with volumetric measurements. AJNR Am J Neuroradiol 2007;28:965-70

13. Ridha HB, Tozer DJ, Symms MR, et al. Quantitative magnetization transfer imaging in Alzheimer disease. Radiology 2007;244:832-37

14. Kiefer C, Brockhaus L, Cattapan-Ludewig K, et al. Multi-parametric classification of Alzheimer's disease and mild cognitive impairment: the impact of quantitative magnetization transfer imaging. NeuroImage 2009;48:657-67

15. Giulietti G, Bozzali M, Figura V, et al. Quantitative magnetization transfer provides information complementary to grey matter atrophy in Alzheimer's disease brain. NeuroImage 2012;59:1114-22

16. Wiest R, Burren Y, Hauf M, et al. Classification of mild cognitive impairment and Alzheimer disease using model-based MR and magnetization transfer imaging. AJNR Am J Neuroradiol 2013;34: $740-46$

17. Bozzali M, Franceschi M, Falini A, et al. Quantification of tissue damage in $\mathrm{AD}$ using diffusion tensor and magnetization transfer MRI. Neurology 2001;57:1135-37

18. Ropele S, Schmidt R, Enzinger C, et al. Longitudinal magnetization transfer imaging in mild to severe Alzheimer disease. AJNR Am J Neuroradiol 2012;33:570-75

19. Kabani NJ, Sled JG, Shuper A, et al. Regional magnetization transfer ratio changes in mild cognitive impairment. Magn Reson Med 2002;47:143-48

20. Kabani NJ, Sled JG, Chertkow H. Magnetization transfer ratio in mild cognitive impairment and dementia of Alzheimer's type. NeuroImage 2002;15:604-10

21. Draganski B, Ashburner J, Hutton C, et al. Regional specificity of MRI contrast parameter changes in normal ageing revealed by voxel-based quantification (VBQ). NeuroImage 2011;55:1423-34

22. Bracco L, Amaducci L, Pedone D, et al. Italian Multicentre Study on Dementia (SMID): a neuropsychological test battery for assessing Alzheimer's disease. J Psychiatr Res 1990;24:213-26

23. McKhann G, Drachman D, Folstein M, et al. Clinical diagnosis of Alzheimer's disease: report of the NINCDS-ADRDA Work Group under the auspices of Department of Health and Human ServicesTask Force on Alzheimer's disease. Neurology 1984;34:939-44

24. Fazekas F, Chawluk JB, Alavi A, et al. MR signal abnormalities at $\mathbf{1 . 5}$ T in Alzheimer's dementia and normal aging. AJNR Am J Neuroradiol 1987;149:351-56

25. Jenkinson M, Bannister P, Brady JM, et al. Improved optimisation for the robust and accurate linear registration and motion correction of brain images. NeuroImage 2002;17:825-41

26. Jost G, Hähnel S, Heiland S, et al. An automated method for volumetric quantification of magnetization transfer of the brain. Magn Reson Imaging 2002;20:593-97
27. Franke K, Luders E, May A. Brain maturation: predicting individual BrainAGE in children and adolescents using structural MRI. NeuroImage 2012;63:1305-12

28. Ashburner J, Friston KJ. Unified segmentation. NeuroImage 2005;26:839-51

29. Tohka J, Zijdenbos A, Evans A. Fast and robust parameter estimation for statistical partial volume models in brain MRI. NeuroImage 2004;23:84-97

30. Rajapakse JC, Giedd JN, Rapoport JL. Statistical approach to segmentation of single-channel cerebral MR images. IEEE Trans Med Imaging 1997;16:176-86

31. Cuadra MB, Cammoun L, Butz T, et al. Comparison and validation of tissue modelization and statistical classification methods in T1-weighted MR brain images. IEEE Trans Med Imaging 2005;24:1548-65

32. Gaser C. Partial volume segmentation with adaptive maximum a posteriori (MAP) approach. NeuroImage 2009;47:S121

33. Fischl B. FreeSurfer. NeuroImage 2012 62:774-81

34. Witten IH, Frank E, Hall MA. Data Mining: Practical Machine Learning Tools and Techniques, 3rd ed. Burlington, Massachusetts: Margan Kaufmann; 2011

35. Castellani RJ, Rolston RK, Smith MA. Alzheimer disease. Dis Mon 2010;56:484-546

36. Janota I, Mountjoy CQ. Asymmetry of pathology in Alzheimer's disease. J Neurol Neurosurg Psychiatry 1988;51:1011-12

37. Stefanits H, Budka H, Kovacs GG. Asymmetry of neurodegenerative disease-related pathologies: a cautionary note. Acta Neuropathol 2012;123:449-52

38. Derflinger S, Sorg C, Gaser C, et al. Grey-matter atrophy in Alzheimer's disease is asymmetric but not lateralized. $J$ Alzheimers Dis 2011;25:347-57

39. Mitchell AJ, Shiri-Feshki M. Rate of progression of mild cognitive impairment to dementia-meta-analysis of 41 robust inception cohort studies. Acta Psychiatr Scand 2009;119:252-65

40. Petersen RC, Smith GE, Waring SC, et al. Mild cognitive impairment: clinical characterization and outcome. Arch Neurol 1999;56:303-08

41. Pennanen C, Testa C, Laakso MP, et al. A voxel based morphometry study on mild cognitive impairment. J Neurol Neurosurg Psychiatry 2005;76:11-14

42. Kalus P, Slotboom J, Gallinat J, et al. Examining the gateway to the limbic system with diffusion tensor imaging: the perforant pathway in dementia. NeuroImage 2006;30:713-20

43. Han Y, Lui S Kuang W, et al. Anatomical and functional deficits in patients with amnestic mild cognitive impairment. PLoS One 2012;7:e28664

44. Chetelat GB. Landeau, F. Eustache, et al. Using voxel-based morphometry to map the structural changes associated with rapid conversion in MCI: a longitudinal MRI study. NeuroImage 2005;27:934-46

45. Cercignani M, Symms MR, Schmierer K, et al. Three-dimensional quantitative magnetisation transfer imaging of the human brain. NeuroImage 2005;27:436-41 